\title{
Field Evaluation of Novel Fungicides against Late Blight of Potato caused by Phytophthora infestans
}

\author{
H. N. Supriya ${ }^{1 *}$, M. S. Nagaraj ${ }^{1}$ and G. K. Sudarshan ${ }^{2}$ \\ ${ }^{1}$ University of Agricultural Sciences, Bengaluru, India \\ ${ }^{2}$ University of Horticultural Sciences, Bagalkot, Karnataka, India \\ *Corresponding author
}

\section{A B S T R A C T}

K e y w or d s
Fungicides,
Late Blight, Potato,
Phytophthora
infestans

\section{Introduction}

Potato, popularly known as the King of Vegetables $^{\text {ee }}$ is a starchy, tuberous crop (Solanum tuberosum L.), belonging to family Solanaceae. It originated from South America, in the mountains of southern Peru and Bolivia. It is the world's fourth-largest food crop, following rice, wheat, and maize. Potato is a valuable source of minerals such as potassium, magnesium and phosphorus and also of dietary antioxidants. A high content of vitamin enhances iron absorption, and high vitamin C / high iron potato varieties could significantly contribute to achieve the daily vitamin $\mathrm{C}$ and iron requirements (Lutaladlo and Castaldi, 2009).

More than 175 diseases/disorders of potato have been short listed by Wellman (1972). Shekhawat (1990) reported about 24 disease or disorders of potato from India. Potato crop is sensitive to many biotic and abiotic stresses and affected by many pests and pathogens (such as fungi, viruses, bacteria and nematode and several insects) which act as main constraints in potato cultivation and destabilize the annual production. Among the diseases, late blight (Phytophthora infestans), early blight (Alternaria solani), bacterial wilt 
(Ralstonia solanacearum), potato viruses etc., are major constraints in different area.

Late blight of potato, caused by Phytophthora infestans (Mont.) de Bary, is one of the serious threats to potato growers throughout world. It is one of the classic examples for pandemic disease of crops. With the introduction of late blight fungus in Europe, staple food of Europeans was destroyed, ultimately causing the devastating famine of history "the Irish famine" in 1845. In India, late blight of potato is one of the important fungal diseases which appear in every year, in serious form. Disease development is favoured by cool, moist weather.

The late blight disease of potato is being becoming an endemic disease in Hassan district in recent years. The disease is most commonly managed by the use of fungicides by the farmer's. But now a day's farmers are not able to save the crop even by spraying the fungicides because, the unscientific use of fungicides such as selection of effective fungicide, time of application, method of application, dose of application and schedule of fungicides.

Hence, scheduling of new fungicides for management of late blight of potato was taken in the present investigation. The best management fungicide schedule methods will be helpful to the potato growing farmers of the region.

\section{Materials and Methods}

A field experiment was laid out in Agricultural Research Station, Gunjevu, during Kharif-2019. The crop was raised with standard cultivation practices of Hassan region. The experiment was performed with Randomized Complete Block Design (RCBD) with three replications and 11 treatments. Entire experimental plot was allowed for natural build of disease on highly susceptible potato cv. Kufri Jyothi. The efficacy novel fungicides against the growth of $P$. infestans tested under in vitro conditions were carried to field experiments. The fungicides were tested as 4 sequential sprays at an interval of 7 days. The first spray was carried out as soon as the first symptom of late blight was noticed.

The experimental site was geographically situated in the Southern transitional zone (Zone- 7) of Karnataka and the place, Hassan, considered as 'hot spot' for late blight of potato is located at $12^{\circ} \mathrm{N} ; 76^{\circ} \mathrm{E} ; 695 \mathrm{MSL}$ elevations and the average rainfall is around $705 \mathrm{~mm} /$ year.

The observations were recorded on germination percentage at 30 and 45DAS. The treatments were imposed by foliar application of fungicides in sequence from 30 DAS at weekly intervals for four times.

The observations were recorded on per cent disease severity before each application during the experimentation period. Observation on disease severity of foliage was recorded by using 1-9 scale as given by CIP (1997) and per cent disease index (PDI) was worked out using formula of wheeler1969.

The vegetative parameters viz., plant height, number of branches per plant, number of leaves per plant were recorded twice at regular peak growth period, Whereas, the yield parameters were recorded at the end of crop period by selecting five random plants per replicated plot in each treatment and mean yield data per hectare was calculated and expressed in tonnes.

CIP scale for recording the field observation on potato late blight: 


\begin{tabular}{|c|c|}
\hline \% area infected & Scale \\
\hline 0 & 1 \\
\hline Up to 3 & 2 \\
\hline Up to 10 & 3 \\
\hline Up to 25 & 4 \\
\hline Up to 50 & 5 \\
\hline Up to 75 & 6 \\
\hline Up to 90 & 7 \\
\hline Up to 99 & 8 \\
\hline Up to 100 & 9 \\
\hline
\end{tabular}

The disease severity was calculated by using the following formula

$$
\text { Per cent disease severity }=\frac{\text { Summation of numerical rating }}{\text { No of plants examined x Maximum disease score }}
$$

Area under disease progress curve (AUDPC)

Area under disease progress curve is an important parameter being frequently used in analytical epidemiology. This is being used in place of disease severity for crop yield forecasters. AUDPC has advantage as it accounts both for disease severity as well as duration of disease development. Both these factors together are closely (positively) related to amount of damage and crop yield losses.

The pattern of an epidemic in terms of the number of lesions or the amount of disease tissue is given by a curve, that shows that progress of the disease over time in a cropped area is called area under disease progress curve, it was calculated by following formula (Shanner and Finey, 1997),

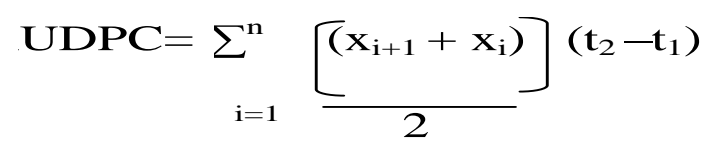

$\mathrm{Xi}=$ Disease severity at time $\mathrm{t}_{\mathrm{i}}$

$\mathrm{X}_{\mathrm{i}+1}=$ Disease severity at time $\mathrm{t}_{\mathrm{i}+1}$

$\mathrm{n}=$ Number of observation

\section{Statistical analysis}

The data so generated from different experiments was analysed using WASP software developed by ICAR- central coastal Agriculture Research Institute, Goa and the inferences were drawn at one and five per cent probability for the laboratory and field experiments respectively.

\section{Results and Discussion}

Results pertaining to the field evaluation of novel fungicides for scheduling on management of late blight disease revealed that spray of Fenamidone $10 \%+$ Mancozeb $50 \%$ WG proved most potent fungicide which recorded $18.00 \%$ of disease severity. Followed by Dimethomorph 50\% WP recorded 24.36 per cent disease severity, Famoxadone 16.6\% + Cymoxanil 22\% SC and Metiram 55\% + Pyroclostrobin 5\% WG recorded 27.68 and 28.63 per cent disease severity which were on par with each other. Among all the treatments Mandipropamid $23.4 \%$ SC and Azoxystrobin $11 \%+$ Tebuconazole $18.3 \%$ SC recorded 38.52 and 37.87 per cent disease severity were found least significant over other treatments. All the treatments were significantly superior over the control (94.04\%) at final observation 70DAP (Table 1, Fig. 1, and Plate 1 and 2). Similar results are in confirmation with studies of Anjaneya Reddy et al., (2015), studied field experiments at Hogalagere, Kolar to test the efficacy of new fungicides using the cultivar Kufri Jyothi. Among the different fungicides tested, Azoxystrobin 23\% $\mathrm{SC} @ 1 \mathrm{~mL}$ per litre is most effective with average Per cent disease index of 13.63 and with the yield of $127.70 \mathrm{q} / \mathrm{ha}$, followed by Fenamidone + Mancozeb $-4 \mathrm{~g}$ per litre with Per cent disease index of 24.50 and with the yield of 119.05 q/ha. Similar results were reported by Nagaraj et al., (2011) and Amin et al., (2013). 
Table.1 Field evaluation on efficacy of different fungicide on disease incidence during Kharif-2019

\begin{tabular}{|c|c|c|c|c|c|c|c|}
\hline Treatment Details & $\begin{array}{c}\text { Dosage } \\
(/ \mathrm{L})\end{array}$ & 35DAP & 42DAP & 49DAP & 56DAP & 63DAP & 70DAP \\
\hline Mancozeb 75\% WP & $2.5 \mathrm{~g}$ & $\begin{array}{c}3.30 \\
(10.43)\end{array}$ & $\begin{array}{c}9.59 \\
(17.92)\end{array}$ & $\begin{array}{c}16.79 \\
(24.18)\end{array}$ & $\begin{array}{c}24.34 \\
(29.56)\end{array}$ & $\begin{array}{c}31.41 \\
(33.97)\end{array}$ & $\begin{array}{c}36.15 \\
(39.96)\end{array}$ \\
\hline Mandipropamid $23.4 \%$ SC & $0.8 \mathrm{~mL}$ & $\begin{array}{c}3.43 \\
(10.47)\end{array}$ & $\begin{array}{c}5.45 \\
(13.37)\end{array}$ & $\begin{array}{l}13.08 \\
(21.18)\end{array}$ & $\begin{array}{c}20.51 \\
(26.92)\end{array}$ & $\begin{array}{c}27.54 \\
(31.65)\end{array}$ & $\begin{array}{c}38.52 \\
(38.36)\end{array}$ \\
\hline Cymoxanil 8\% + Mancozeb $72 \%$ & $3.0 \mathrm{~g}$ & $\begin{array}{c}2.00 \\
(8.13)\end{array}$ & $\begin{array}{c}7.98 \\
(16.05)\end{array}$ & $\begin{array}{l}14.28 \\
(22.16)\end{array}$ & $\begin{array}{l}20.18 \\
(26.68)\end{array}$ & $\begin{array}{c}26.05 \\
(30.69)\end{array}$ & $\begin{array}{l}30.25 \\
(33.37)\end{array}$ \\
\hline Dimethomorph 50\% WP & $1.0 \mathrm{~g}$ & $\begin{array}{c}1.50 \\
(6.96)\end{array}$ & $\begin{array}{l}11.88 \\
(20.11)\end{array}$ & $\begin{array}{c}16.46 \\
(23.93)\end{array}$ & $\begin{array}{l}21.47 \\
(27.60)\end{array}$ & $\begin{array}{c}26.29 \\
(30.84)\end{array}$ & $\begin{array}{c}24.39 \\
(29.59)\end{array}$ \\
\hline Fenamidone $10 \%$ + Mancozeb $50 \%$ & $4.0 \mathrm{~g}$ & $\begin{array}{c}1.25 \\
(5.34)\end{array}$ & $\begin{array}{c}4.45 \\
(11.97)\end{array}$ & $\begin{array}{c}8.49 \\
(16.93)\end{array}$ & $\begin{array}{l}11.70 \\
(20.00)\end{array}$ & $\begin{array}{c}14.80 \\
(22.62)\end{array}$ & $\begin{array}{l}18.00 \\
(25.09)\end{array}$ \\
\hline $\begin{array}{l}\text { Famoxadone } 16.6 \% \text { + Cymoxanil } \\
22 \%\end{array}$ & $1.0 \mathrm{~mL}$ & $\begin{array}{c}1.67 \\
(7.33)\end{array}$ & $\begin{array}{c}7.08 \\
(15.36)\end{array}$ & $\begin{array}{l}12.80 \\
20.96 \\
\end{array}$ & $\begin{array}{c}17.78 \\
(24.93)\end{array}$ & $\begin{array}{c}23.87 \\
(29.24)\end{array}$ & $\begin{array}{c}27.68 \\
(31.74)\end{array}$ \\
\hline $\begin{array}{l}\text { Boscalid 25.2\% + Pyraclostrobin } \\
2.8 \%\end{array}$ & $2.0 \mathrm{~g}$ & $\begin{array}{c}2.33 \\
(8.74)\end{array}$ & $\begin{array}{l}12.76 \\
(20.91)\end{array}$ & $\begin{array}{l}18.73 \\
(25.64)\end{array}$ & $\begin{array}{c}24.82 \\
(29.87)\end{array}$ & $\begin{array}{c}28.61 \\
(32.33)\end{array}$ & $\begin{array}{c}30.57 \\
(33.56)\end{array}$ \\
\hline $\begin{array}{l}\text { Azoxystrobin } 11 \% \text { + Tebuconazole } \\
18.3 \%\end{array}$ & $1.0 \mathrm{~mL}$ & $\begin{array}{c}4.10 \\
(11.65)\end{array}$ & $\begin{array}{c}9.60 \\
(17.96)\end{array}$ & $\begin{array}{c}17.32 \\
(24.86)\end{array}$ & $\begin{array}{l}23.56 \\
(28.24)\end{array}$ & $\begin{array}{c}32.74 \\
(34.21)\end{array}$ & $\begin{array}{c}37.87 \\
(37.98)\end{array}$ \\
\hline Metiram 55\% + Pyraclostrobin 5\% & $2.1 \mathrm{~g}$ & $\begin{array}{c}1.97 \\
(7.89)\end{array}$ & $\begin{array}{c}10.91 \\
(19.21)\end{array}$ & $\begin{array}{c}16.17 \\
(23.71)\end{array}$ & $\begin{array}{c}22.08 \\
(28.02)\end{array}$ & $\begin{array}{c}27.50 \\
(31.62)\end{array}$ & $\begin{array}{c}28.63 \\
(32.35)\end{array}$ \\
\hline $\begin{array}{l}\text { Flupicolide } 4.44 \% \text { + Fosetyl-Al } \\
66.6 \%\end{array}$ & $3.0 \mathrm{~g}$ & $\begin{array}{c}3.22 \\
(10.01)\end{array}$ & $\begin{array}{c}8.83 \\
(17.27)\end{array}$ & $\begin{array}{l}15.38 \\
(23.08)\end{array}$ & $\begin{array}{c}22.32 \\
(28.18)\end{array}$ & $\begin{array}{c}28.91 \\
(32.52)\end{array}$ & $\begin{array}{c}33.26 \\
(35.22)\end{array}$ \\
\hline Untreated Control & -- & $\begin{array}{c}5.60 \\
(13.65)\end{array}$ & $\begin{array}{c}15.19 \\
(22.92)\end{array}$ & $\begin{array}{c}34.39 \\
(35.90)\end{array}$ & $\begin{array}{c}53.30 \\
(46.89)\end{array}$ & $\begin{array}{c}72.06 \\
(58.11)\end{array}$ & $\begin{array}{c}94.04 \\
(75.89)\end{array}$ \\
\hline \multicolumn{2}{|l|}{ S.Em \pm} & 0.52 & 0.85 & 0.67 & 0.63 & 0.61 & 0.63 \\
\hline \multicolumn{2}{|l|}{ CD@ 5\% } & 3.15 & 2.54 & 1.62 & 1.29 & 1.16 & 1.26 \\
\hline
\end{tabular}

Note: Figures in the parenthesis are arc sine transformed values 
Table.2 Effect of Fungicide Scheduling on AUDPC (Area under disease progress curve) during Kharif-2019

\begin{tabular}{|c|c|c|c|c|c|c|}
\hline Treatments & 35-42DAP & 42-49 DAP & 49-56 DAP & 56-63DAP & 63-70 DAP & 70-77 DAP \\
\hline Mancozeb $75 \%$ WP & 11.55 & 45.11 & 92.33 & 143.95 & 195.12 & 236.46 \\
\hline Mandipropamid $23.4 \%$ SC & 11.90 & 30.97 & 64.85 & 117.56 & 168.17 & 231.21 \\
\hline Cymoxanil 8\% + Mancozeb $72 \%$ & 7.00 & 34.93 & 77.91 & 120.61 & 161.80 & 197.05 \\
\hline Dimethomorph 50\% WP & 5.25 & 46.83 & 99.19 & 132.75 & 167.16 & 177.39 \\
\hline Fenamidone $10 \%$ + Mancozeb $50 \%$ & 4.37 & 19.95 & 45.29 & 70.66 & 92.75 & 114.80 \\
\hline Famoxadone $16.6 \%$ + Cymoxanil $22 \%$ & 5.84 & 30.62 & 69.58 & 107.03 & 145.77 & 180.42 \\
\hline Boscalid 25.2\% + Pyraclostrobin $2.8 \%$ & 8.15 & 52.81 & 110.21 & 152.42 & 187.00 & 207.13 \\
\hline Azoxystrobin $11 \%$ + Tebuconazole $18.3 \%$ & 14.35 & 47.95 & 94.22 & 143.08 & 197.05 & 247.13 \\
\hline Metiram 55\% + Pyraclostrobin 5\% & 6.89 & 45.08 & 94.78 & 133.87 & 173.53 & 196.45 \\
\hline Flupicolide $4.44 \%$ + Fosetyl-Al $66.6 \%$ & 11.27 & 42.17 & 84.73 & 131.95 & 179.30 & 217.59 \\
\hline Untreated Control & 19.60 & 72.76 & 173.53 & 306.91 & 438.76 & 581.35 \\
\hline S.Em \pm & 0.29 & 0.44 & 0.31 & 0.28 & 0.27 & 0.34 \\
\hline CD@5\% & 0.85 & 1.31 & 0.92 & 0.84 & 0.81 & 1.01 \\
\hline
\end{tabular}

Table.3 Effect of fungicides against late blight of potato on vegetative parameters during Kharif-2019

\begin{tabular}{|c|c|c|c|c|c|c|c|c|}
\hline \multirow{2}{*}{$\begin{array}{l}\text { Treatments } \\
\text { Mancozeb } 75 \% \mathrm{WP}\end{array}$} & \multicolumn{2}{|c|}{$\%$ Emergence } & \multicolumn{2}{|c|}{ Plant height(cm) } & \multicolumn{2}{|c|}{$\begin{array}{c}\text { No. of haulms per } \\
\text { plant }\end{array}$} & \multicolumn{2}{|c|}{$\begin{array}{c}\text { Number of leaves per } \\
\text { plant }\end{array}$} \\
\hline & 30DAP & 45DAP & 30DAS & 60DAS & 30DAS & 60DAS & 30DAS & 60DAS \\
\hline Mandipropamid $23.4 \%$ SC & 87.19 & 91.24 & 30.46 & 50.53 & 2.10 & 4.83 & 42.57 & 84.57 \\
\hline Cymoxanil 8\% + Mancozeb $72 \%$ & 89.51 & 92.92 & 25.63 & 45.63 & 2.10 & 4.7 & 41.23 & 83.23 \\
\hline Dimethomorph 50\% WP & 88.35 & 93.70 & 37.00 & 55.67 & 2.27 & 5.63 & 45.40 & 87.40 \\
\hline Fenamidone $10 \%$ + Mancozeb $50 \%$ & 85.36 & 91.99 & 42.33 & 64.00 & 2.53 & 6.53 & 51.90 & 93.90 \\
\hline Famoxadone $16.6 \%$ + Cymoxanil $22 \%$ & 90.42 & 96.57 & 44.64 & 64.66 & 2.53 & 7.33 & 57.40 & 99.40 \\
\hline Boscalid 25.2\% + Pyraclostrobin $2.8 \%$ & 91.44 & 93.73 & 42.33 & 62.66 & 2.51 & 6.16 & 51.00 & 93.00 \\
\hline Azoxystrobin $11 \%$ + Tebuconazole $18.3 \%$ & 86.88 & 91.69 & 32.83 & 55.40 & 2.27 & 4.96 & 44.97 & 86.97 \\
\hline Metiram 55\% + Pyraclostrobin 5\% & 82.45 & 95.48 & 22.23 & 43.56 & 1.93 & 4.7 & 38.70 & 80.70 \\
\hline Flupicolide $4.44 \%$ + Fosetyl-Al $66.6 \%$ & 85.71 & 92.63 & 41.67 & 61.66 & 2.27 & 6.00 & 45.60 & 87.60 \\
\hline Untreated Control & 84.05 & 91.83 & 31.63 & 51.36 & 2.17 & 4.86 & 44.50 & 86.50 \\
\hline S.Em \pm & 89.37 & 94.49 & 21.73 & 43.00 & 1.80 & 4.63 & 38.33 & 80.33 \\
\hline CD@ 5\% & 2.59 & 1.49 & 2.27 & 2.60 & 0.08 & 0.60 & 3.57 & 0.91 \\
\hline
\end{tabular}


Table.4 Effect of fungicides against late blight of potato on yield parameters during Kharif-2019

\begin{tabular}{|c|c|c|c|c|c|}
\hline \multirow[t]{2}{*}{ Treatment Details } & \multicolumn{5}{|c|}{ Yield parameters } \\
\hline & $\begin{array}{c}\text { Number of } \\
\text { tubers per } \\
\text { plant }\end{array}$ & $\begin{array}{c}\text { Per cent } \\
\text { increase } \\
\text { over control }\end{array}$ & $\begin{array}{l}\text { Tuber Yield } \\
\text { per plot } \\
\text { (kg) }\end{array}$ & $\begin{array}{c}\text { Tuber Yield } \\
\text { (t/ha) }\end{array}$ & $\begin{array}{c}\text { Per cent } \\
\text { increase } \\
\text { over control }\end{array}$ \\
\hline Mancozeb $75 \%$ WP & 4.12 & 04.00 & 10.72 & 10.47 & 34.23 \\
\hline Mandipropamid $23.4 \%$ SC & 4.10 & 03.33 & 10.16 & 9.93 & 27.30 \\
\hline Cymoxanil 8\% + Mancozeb 72\% & 4.67 & 22.33 & 13.99 & 13.67 & 75.25 \\
\hline Dimethomorph 50\% WP & 6.00 & 66.66 & 15.05 & 14.70 & 88.46 \\
\hline Fenamidone 10\% + Mancozeb 50\% & 6.63 & 87.66 & 15.49 & 15.13 & 93.97 \\
\hline Famoxadone $16.6 \%$ + Cymoxanil $22 \%$ & 5.33 & 44.33 & 14.87 & 14.53 & 86.28 \\
\hline Boscalid 25.2\% + Pyraclostrobin 2.8\% & 4.33 & 11.00 & 13.24 & 12.93 & 65.76 \\
\hline Azoxystrobin $11 \%$ + Tebuconazole $18.3 \%$ & 4.10 & 03.33 & 10.13 & 09.90 & 26.92 \\
\hline Metiram 55\% + Pyraclostrobin 5\% & 5.00 & 33.33 & 14.33 & 14.04 & 80.00 \\
\hline Flupicolide $4.44 \%$ + Fosetyl-Al $66.6 \%$ & 4.33 & 11.00 & 12.42 & 12.13 & 55.51 \\
\hline Untreated Control & 4.00 & 00.00 & 07.16 & 07.80 & 00.00 \\
\hline S.Em \pm & 0.63 & - & 0.21 & 0.23 & - \\
\hline CD @ 5\% & 1.85 & - & 0.63 & 0.69 & - \\
\hline
\end{tabular}


Fig.1 Graphical representation of efficiency of novel fungicide schedules on disease severity during Kharif-2019

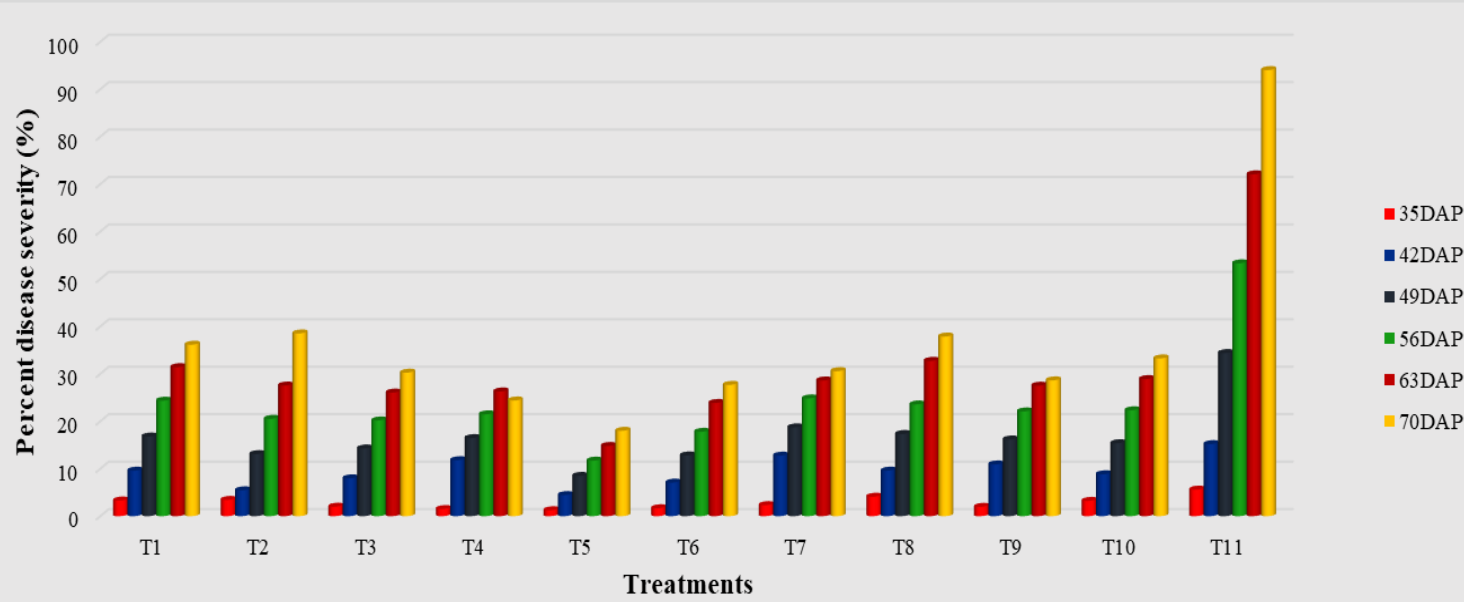

T1- Mancozeb 75\% WP

T2- Mandipropamid 23.4\% SC

T3- Cymoxanil 8\% + Mancozeb 72\%

T4- Dimethomorph 50\% WP

T5- Fenamidone 10\% + Mancozeb 50\%

T6- Famoxadone $16.6 \%+$ Cymoxanil $22 \%$
T7- Boscalid 25.2\% + Pyraclostrobin $2.8 \%$

T8- Azoxystrobin 11\% + Tebuconazole $18.3 \%$

T9- Metiram 55\% + Pyraclostrobin 5\%

T10- Flupicolide $4.44 \%$ + Fosetyl-Al $66.6 \%$

T11- Untreated Control

Fig.2 Graphical representation of efficiency of different fungicide schedules on AUDPC during Kharif-2019

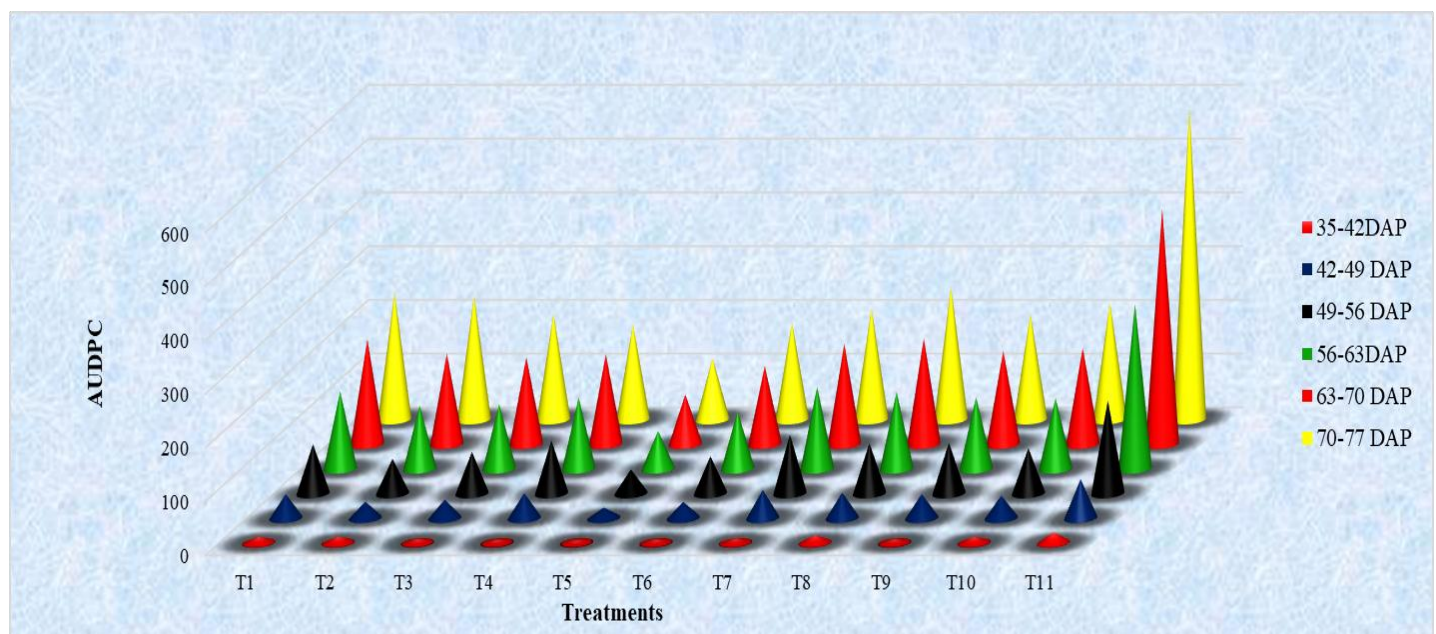

T1- Mancozeb 75\% WP

T2- Mandipropamid 23.4\% SC

T3- Cymoxanil 8\% + Mancozeb 72\%

T4- Dimethomorph 50\% WP

T5- Fenamidone 10\% + Mancozeb 50\%

T6- Famoxadone 16.6\% + Cymoxanil 22\%
T7- Boscalid 25.2\% + Pyraclostrobin $2.8 \%$

T8- Azoxystrobin 11\% + Tebuconazole $18.3 \%$

T9- Metiram 55\% + Pyraclostrobin 5\%

T10- Flupicolide 4.44\% + Fosetyl-Al 66.6\%

T11- Untreated Control 
Fig.3 Graphical representation of influence of different fungicide schedules on tuber yield of Potato during Kharif-2019

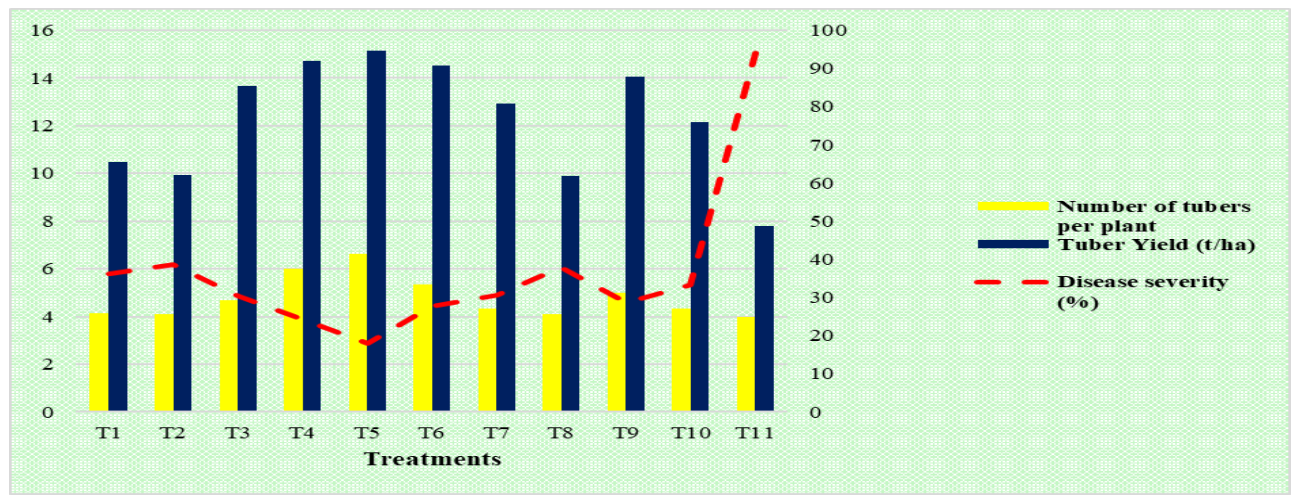

Plate.1 Comparison of treatments $\mathrm{T}_{5}, \mathrm{~T}_{4}$ and $\mathrm{T}_{6}$ plots with control at 52DAP

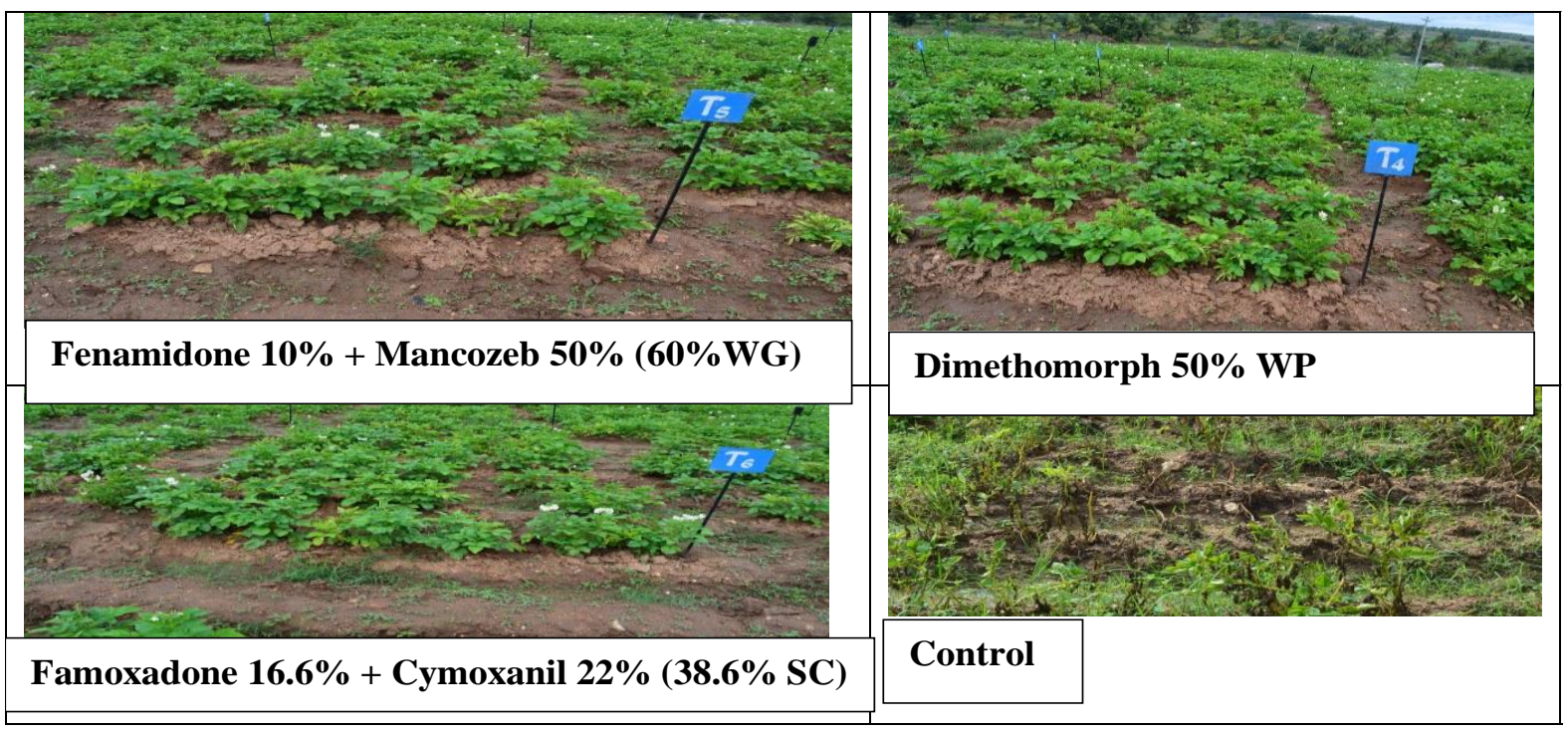

Plate.2 Overall view of potato field raised to carried out experiment on testing efficacy new fungicides on potato late blight

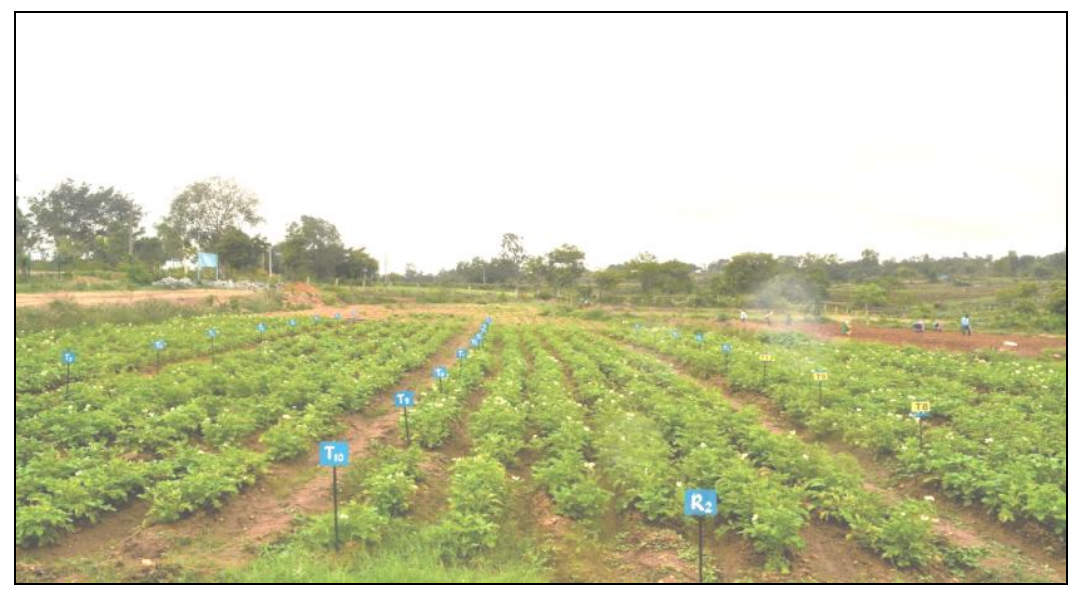


Lowest value of AUDPC was documented in Fenamidone $10 \%$ + Mancozeb $50 \% \mathrm{WG}$ of 114.80 followed by Dimethomorph 50\% WP of 117.39. The recorded AUDPC increased with time, further rate of increasing AUDPC values is highest in followed by treatment nd lowest rate of increase of AUDPC values recorded in and (Table 2 and Fig. 2). These results are in line of work done by Khadka et al., (2016) carried out the field experiments in two consecutive years 2014-15 and 2015-16 at Khajura, Nepal to evaluate the efficacy of eight fungicides viz. Realbet (Dimethomorph $50 \%$ WP), Sectin (Fenamidone 10\% + Mancozeb 50\%WG), Indofil M-45 (Mancozeb $\quad 75 \% \quad$ WP), Prtotector (Chlorothalonil 70\% WP), Galaxy (Metalaxyl $35 \%$ WS) and Copper oxychloride for the management of potato late blight, Phytophthora infestans (Mont) de Bary. The results shows that lowest AUDPC and rAUDPC was recorded in Realbet (Dimethomorph 50\%) and Sectin (Fenamidone 10\% + Mancozeb 50\%WG) among the tested fungicide while highest yield per ha was also recorded in these fungicide.

Treatment Fenamidone $10 \%+$ Mancozeb $50 \% \mathrm{WG}$ recorded maximum plant height of $64.66 \mathrm{~cm}$ as compared to all treatments and found on par with Dimethomorph 50\% WP $(64.00 \mathrm{~cm})$ followed by Famoxadone $16.6 \%+$ Cymoxanil $22 \% \mathrm{SC}(62.66 \mathrm{~cm})$ at 60 DAP.

Maximum number of haulms per potato plant of 7.33 was documented in Fenamidone $10 \%$ + Mancozeb 50\%WG found significant among all treatments followed by Dimethomorph 50\% WP of 6.53 found on par with Famoxadone 16.6\% + Cymoxanil 22\% SC of 6.16, while control recorded the lowest number of plant haulms (4.63).

Maximum number of leaves 99.40 were reported in Fenamidone $10 \%+$ Mancozeb
$50 \% \mathrm{WG}$ at 60 DAP fond substantially superior with other treatments, followed by Dimethomorph 50\% WP of 93.90 found on par with Famoxadone 16.6\% + Cymoxanil $22 \%$ SC of 93.00 , while the minimum number of leaves were observed in control (80.33) (Table 3).

Maximum number of 6.63 tuber per plant was obtained in Fenamidone 10\% + Mancozeb $50 \%$ WG which found on par with Dimethomorph 50\% WP (6.00) were highly effective over other. Whereas control plot recorded least number of tubers (4.00) per plant. Maximum tuber yield per hectare was obtained in Fenamidone 10\% + Mancozeb $50 \%$ WG (15.13 t/ha) followed by Dimethomorph 50\% WP (14.70 t/ha) found on par with Famoxadone $16.6 \%+$ Cymoxanil $22 \%$ SC $(14.53 \mathrm{t} / \mathrm{ha})$ whereas, in control treatment recorded lowest yield of $7.80 \mathrm{t} / \mathrm{ha}$ and found inferior to all other treatments (Table 4 and Fig. 3). The results are in agreement with findings of Kavya (2016) conducted field study to evaluate different fungicides and result revealed that, the treatment Fenamidone 10\% + Mancozeb 50\% found to be highly effective in managing late blight of potato with least percent disease severity of 16.67 and highest yield of 28.42 t/ha. Whereas, the control treatment recorded $100 \%$ disease severity with lowest yield of 7.12 t/ha. Similar results also obtained by Harisha (2017).

\section{References}

Amin, M., Mulugeta, N. and Selvaraj, T., 2013. Field evaluation of new fungicide, Victory 72 WP for management of potato and tomato late blight (Phytophthora infestans (Mont) de Bary) in West Shewa Highland, Oromia, Ethiopia. J. Plant Pathol., 4:192.

Anjaneya Reddy, B., Sreenath, A Rekha, D. 
and Devappa, V., 2015. Field efficacy of new fungicide molecules against late blight of potato in Southern Karnataka. Abstr. $3^{\text {rd }}$ International Symposium on Phytophthora: Taxonomy, Genomics, Pathogenicity, Resistance and Disease Management. 54pp.

CIP (1997) Annual Report. The International Potato Centre, Lima Peru: 179p.

Harisha, Y. N., 2017. Studies on management of late blight disease of potato caused by Phytophthora infestans (Mont.) de Bary. M. Sc. (Agri) Thesis. University of Agricultural Sciences, Bengaluru.

Hawkes, J. C. 1945. The story of potato. Discovery, $2 \mathrm{p}$.

Kavya, B., 2016. Studies on the late blight disease of potato (solanum tuberosum L.). M. Sc. (Agri) Thesis. University of Agricultural Sciences, Bengaluru.

Khadka, R. B., Rawal, R., Marasaini, M., Shrestha, G. K., Chapagain, T. and Gautam, I. P., 2016. Evaluation of Different Fungicides and Clones against Potato Late Blight (Phytophthora infestans) In Western Nepal. 5th International Conference on Agriculture, Environment and Biological Sciences (ICAEBS-16).
Lutaladlo, N. B. and Castaldi, L. 2009. Potato: The hidden treasure. International Year of the Potato. 22(6): 491-493.

Nagaraj, M. S., Siddagangaiah, Joseph, T. A. and Vijayalakshman, M., 2011. Survey on the epiphytotics of late blight on potato in Hassan and Chikmagalore districts of Karnataka during 2009-10. Abstr.64 ${ }^{\text {th }}$ Indian Phytopathological Society Annual meeting and National symposium on Biology of infection, immunity and disease control in pathogen-plant interactions. $17 \mathrm{pp}$.

Shanner, G. and Finey, R E., 1997. The effect of nitrogen fertilizer on the expression of slow mildewing resistance in lenox wheat. Phytopathol., 67: 1051-1056.

Shekhawat, G. S. 2000. Management of potato diseases through host resistance. J. Mycol. Pl. Pathol. 30(2): 143-150.

Shekhawat, G. S. 1990. Potato Diseases and Pests and their Management in the Hills of Himanchal Pradesh. J. Indian Potato Assoc. pp. 94-101.

Wellman, F. L. 1972. Tropical American Plant Disease. Searecrow Press, Metuchen, New Jersey.

\section{How to cite this article:}

Supriya, H. N., M. S. Nagaraj and Sudarshan, G. K. 2020. Field Evaluation of Novel Fungicides against Late Blight of Potato caused by Phytophthora infestans. Int.J.Curr.Microbiol.App.Sci. 9(11): 1759-1768. doi: https://doi.org/10.20546/ijcmas.2020.911.208 